\title{
Nerve function in workers with long term exposure to trichloroethene
}

\author{
Marc W M M Ruijten, Maarten M Verberk, Herman J A Sallé
}

\begin{abstract}
Certain functions of the nervous system were examined in 31 printing workers (mean age 44) exposed to trichloroethene (mean duration 16 years) and 28 controls (mean age 45 ). In the sural nerve the conduction velocity (SNCV), response amplitude, and refractory period (SRP) were measured. The latencies of the masseter and the blink reflex were determined to test the trigeminal nerve. In the peroneal nerve the conduction velocity of fast and slow nerve fibres, the response amplitude, and the refractory period were determined. As a measure of autonomic nerve function the response of the heart rate was determined to isometric muscle contraction and deep breathing. Individual cumulative exposure was calculated on the basis of exposure levels in the past. The mean cumulative exposure of the exposed workers was 704 ppm $\times$ years. For the assessment of the exposure effect relation a multiple linear regression model was used. A slight reduction $(-1 \cdot 1 \mathrm{~m} / \mathrm{s})$ in the SNCV was found and a prolongation ( $0.4 \mathrm{~ms})$ of the SRP (mean of the controls $1.95 \mathrm{~ms}$ ). The latency of the masseter reflex (mean $10.4 \mathrm{~ms}$ ) had increased $(0.4 \mathrm{~ms})$. With respect to the blink reflex no prolongation was found. No impairment was found in the functions of motor and autonomic nerves. This study shows that the refractory period may be a sensitive indicator of preclinical toxic neuropathies. Long term exposure to trichloroethene at threshold limit values (about $35 \mathrm{ppm}$ ) may slightly affect the trigeminal and sural nerves.
\end{abstract}

Trichloroethene (TRI) has been applied in industry as a solvent and a degreasing agent since the beginning of this century. In medicine it was used as an

Coronel Laboratory, Faculty of Medicine, University of Amsterdam, The Netherlands

MW M M Ruijten, M M Verberk, H J A Sallé anaesthetic and analgesic. The neurotoxicity of TRI has been established in both applications. ${ }^{123}$ Lesions of the cranial nerves, the trigeminal nerve in particular, were found after very high exposures.

Several reports suggest that non-accidental industrial exposure to TRI may also result in some nerve impairment. ${ }^{4567}$ These reports do not allow the derivation of exposure effect relations. This hampers the evaluation of the threshold limit value with respect to possible neurotoxic effects. In a review, Spencer and Schaumburg also state that there are no adequate epidemiological studies of worker populations to evaluate the neurotoxicity of TRI. ${ }^{8}$

Several methods have been proposed that may contribute to the early detection of TRI induced nerve dysfunctions. Matikainen and Juntunen ${ }^{9}$ studied the variation in heart rate in workers with a toxic neuropathy to ascertain the function of the autonomic nerve system. Studies in diabetic and uraemic patients suggest that a prolongation of the refractory period of nerve fibres may precede a decrease in the conventionally measured conduction velocity. ${ }^{1011}$ In workers exposed to $\mathrm{CS}_{2}$ we found slight nerve dysfunction when applying these two methods. ${ }^{12}$ It is well known that solvents may also affect central nervous functions, resulting in psychoorganic disturbances. Hogstedt $e t a l^{13}$ proposed that the so called questionnaire- 16 be used for a first crude screening of neuropsychiatric symptoms.

Our investigation had two objectives. The first was to determine whether exposure to TRI at about the threshold limit value affects the peripheral nerve system and the trigeminal nerve in particular and to screen for symptoms of the central nervous system with the questionnaire-16. The second was to study the feasibility of the proposed diagnostic methods.

\section{Subjects}

Male printers who had been exposed to TRI for six years or more as well as several suitable non-exposed workers from the same printing works were personally contacted by local occupational health personnel; 68 workers agreed to participate. After exclusion of workers with other risk factors for neuropathy or an alcohol consumption of more than 50 glasses a week, 
the analyses concerned 31 exposed workers and 28 controls. The exposed workers had a mean age of 44 (SD 9) and had been exposed during 16 (SD 9) years (range 6-37 years). The controls were matched on a group basis with the exposed workers with respect to physical job activity, education, nationality, and age (mean 45 (SD 9)); they had all been employed for at least six years in the print works, but had never been exposed occupationally to TRI. Several had been exposed occasionally (less than one hour a week) to terpentine-like organic solvents used as cleaning agents. Both groups consisted, in descending order of frequency, of Dutch, Turkish, Spanish, and Italian nationals.

\section{Exposure}

All exposed subjects worked in the printing process. Up to three years before the investigation all printing was with ink that contained TRI as solvent. No exposure to neurotoxicants other than TRI occurred. During the past three years an increasing part of the printing was carried out in a new location with a water based printing ink. At the time of the investigation the process with TRI was only used about half of the time on a reduced number of presses. Hence monitoring at the time of the investigation would not yield a meaningful measure of exposure; we had to rely on exposure data from monitoring activities in the past conducted by plant personnel with gas detection tubes...In 1966 about 100 samples were taken on 16 consecutive working days on defined locations; the mean concentration of TRI was about $80 \mathrm{ppm}$. In 1976 about 90 samples were taken and showed a mean concentration of 70 ppm. After these measurements local exhausts were installed. Later measurements in 1976 and 1981 showed a mean concentration of $35 \mathrm{ppm}$.

Apart from the installation of the local exhaust and the half time printing with TRI-based ink during the past three years no significant changes in the process had occurred that might have influenced the exposure. The exposure was estimated to be $17 \mathrm{ppm}$ for the past three years, $35 \mathrm{ppm}$ for the preceding eight years (since the installation of the exhaust) and $70 \mathrm{ppm}$ before that. An indication existed for slight differences $(10-20 \%)$ in exposure between jobs. The quality of the exposure data did not permit a differentiation of the exposure with respect to different jobs, however.

Because nervous impairment will probably be due to long term exposure to TRI an individual cumulative exposure $\left(E_{c}\right)$ has been calculated as the sum of the time spent in different exposure periods and the estimated exposure in those periods (ppm $\times$ years). The mean cumulative exposure of the exposed workers was $704 \mathrm{ppm} \times$ years $($ SD 583, range $160-2150 \mathrm{ppm} \times$ years).

\section{Methods}

The examinations were performed in two warm rooms in the health centre at the plant. The investigators did not know the workers' exposure state. After the examination the worker assisted by an investigator completed a general questionnaire concerning drinking habits, diseases, exposure to other factors that might cause neuropathy, and neurological symptoms as well as the questionnaire16. An interpreter was present to help those workers who were not very familiar with the Dutch language. All workers were subjected successively to the following tests.

\section{AUTONOMIC NERVE FUNCTION}

The subject was in a supine position during the examination and during 10 minutes of rest before it. Using chest leads a cardiotachogram was recorded on an ink writer.

For the measurement of the forced respiratory sinus arrhythmia (FRSA) the subject was instructed to perform six consecutive maximal inspirations (five seconds) and expirations (five seconds) in one minute; after two minutes rest one repetition followed. We determined the mean of the differences between the maximum (inspiratory) and minimum (expiratory) instantaneous (beat to beat) heart rate during the second cycle. ${ }^{12}$

For the measurement of the muscle heart reflex (MHR) the subject was asked to exert maximal isometric power on a hand dynamometer during five seconds on verbal command; 4-5 repetitions followed with intervals of about 90 seconds. The grip force was monitored on the ink writer. We calculated the mean of the differences of the maximum instantaneous heart rate during muscle contractions and the resting heart rate on the basis of three trials in which the exerted power was at least $80 \%$ of the individual maximum.

The resting arrhythmia (RA) was determined as the standard deviation of the instantaneous heart rate in the 30 seconds immediately preceding the MHR responses. The mean of the standard deviations of three trials was used to estimate the heart rate variation during rest.

A combined $Z$ score $\left(Z_{\text {aut }}\right)$ was then calculated as being the sum of the individual $Z$ scores of the FRSA and MHR.

\section{TRIGEMINAL NERVE FUNCTION}

The masseter reflex and blink reflex were measured as both concern the trigeminal nerve. The subject remained in the position he had taken for the measurement of autonomic nerve function, holding his head in a position looking straight up with the eyes shut; the mouth was relaxed and closed with the teeth just apart. At least eight reflex responses were amplified, recorded on a storage oscilloscope, and 
photographed. The mean of the three shortest reflex latencies was used as parameter.

The masseter reflex is a myotatic reflex. The response is a bilateral contraction of the masseter muscles; afferent (mandibular root) and efferent (motor root) pathways run through the trigeminal nerve. ${ }^{14}$ Surface electrodes were attached over the right masseter muscle and a rubber roller attached to a thin bar was pressed lightly against the subject's chin. The reflex responses were elicited by unannounced taps with a modified reflex hammer on the rubber roller; upon touching the roller an electric circuit was closed triggering the sweep.

The blink reflex is an exteroceptive reflex. The response is a contraction of the orbicularis oculi muscles consisting of an early olygosynaptic ipsilateral (R1) and a late polysynaptic bilateral response (R2). The afferent pathway runs through the sensory trigeminal nerve, the efferent pathway through the facial nerve. ${ }^{14}$ The responses were elicited by electrical stimulation of the right supraorbital nerve; each stimulus was announced. The active recording electrode was placed on the muscle near the lateral canthus and the reference electrode on the temple. Only the latency of the R1 response was measured because of the better reproducibility and the smaller interindividual differences.

\section{PERIPHERAL NERVE FUNCTION}

The subject's leg was warmed up to the knee in a barrel of water $\left(37^{\circ} \mathrm{C}\right)$ for 10 minutes before electrophysiological testing. During the measurement the skin temperature was kept at about $35^{\circ} \mathrm{C}$ with an infrared heater. The skin temperature close to all electrodes was measured. All stimuli were of $1.2 \times$ current that just gave a maximal response. Only surface electrodes were used.

The motor nerve conduction velocity, (MNCV) of the peroneal nerve and the baseline to negative peak amplitude of the compound muscle action potential (CMAP) were determined by stimulating at the fibular head and the ankle and recording from the extensor digitorum brevis muscle. The motor refractory period (MRP) was determined using a technique adapted from Faisst and Meyer ${ }^{15}$ and Kimura. ${ }^{16}$ In this technique two stimuli (conditioning resp test stimulus) are delivered to the nerve at the proximal site with critical intervals of $0.5-3 \mathrm{~ms}$; about simultaneously with the conditioning stimulus a third stimulus is delivered at the distal site to eliminate the response to the former by antidromic collision. The interstimulus interval (ISI) that would result in a test response with an area of $50 \%\left(\mathrm{MRP}_{50}\right)$ and $80 \%$ $\left(\mathrm{MRP}_{80}\right)$ of a single response following proximal stimulation were used as parameters. The conduction velocity of slow motor fibres (CVSF) was determined with the antidromic collision technique adapted from Hopf ${ }^{1718}$; the ISI that resulted in a response with an amplitude of $80 \%$ was taken as the criterion. From this ISI the $\mathrm{MRP}_{50}$ was subtracted to derive the conduction time of the slow fibres. For details concerning the electrophysiological techniques we refer to Ruijten et al. ${ }^{12}$

The sensory nerve conduction velocity (SNCV) and the peak to peak amplitude of the compound nerve action potential (CNAP) of the sural nerve were determined antidromically by electrical stimulation distal to the lateral head of the gastrocnemius muscle and recording at the lateral malleolus. The SNCV was calculated on the basis of the latency of the first positive peak after averaging of 4-8 responses. As parameters for the sensory refractory period (SRP) we used the ISIs that would result in a response with an amplitude of $50 \%\left(\mathrm{SRP}_{50}\right)$ and $80 \%$ $\left(\mathrm{SRP}_{80}\right)$.

\section{DATA ANALYSIS}

The difference between the exposed workers and the controls was calculated by analysis of covariance (ANCOVA); the effect of cumulative exposure was calculated by multiple linear regression analysis (MLR); the control group was included in each analysis. In each analysis we controlled for age, alcohol consumption, and nationality by including them as covariables. The alcohol consumption was calculated as a cumulative measure (years $\times$ glasses/ week). For the autonomic nerve parameters we included the Quetelet-index and for all peripheral nerve parameters we also included body length (except for refractory periods) and skin temperature. A one sided level of significance of $5 \%$ was used. For non-normal distributed dependent variables a log or square root transformation was used.

\section{Results}

The results of the MLR analyses for the trigeminal reflexes showed an increase in the masseter reflex latency of $0.38 \mathrm{~ms}$ for the mean $\mathrm{E}_{\mathrm{c}}(704 \mathrm{ppm} \times$ years); the mean latency of the controls was $10.4 \mathrm{~ms}$ (table). Because of rather strict criteria for using masseter reflex registrations, the data of 29 subjects (14 exposed workers and 15 controls) were used instead of the total group of 59 . No effects were found for the blink reflex.

In a similar analysis the sural nerve showed an increase in the $S R P_{80}$ of $0.40 \mathrm{~ms}$; the mean of the controls was $1.95 \mathrm{~ms}$. For this nerve a decrease of $1.1 \mathrm{~m} / \mathrm{sec}$ in the SNCV was also found (table). The figure shows the results of the $\mathrm{SRP}_{80}$ after correction for covariables; the results refer to 20 exposed workers and 23 controls.

The motor and autonomic nerve parameters showed no effects related to cumulative exposure; the calculated changes in these nerve functions were too slight to be meaningful.

In comparing the exposed workers with the con- 
Effects of exposure to trichloroethene in 31 printers compared with 28 controls

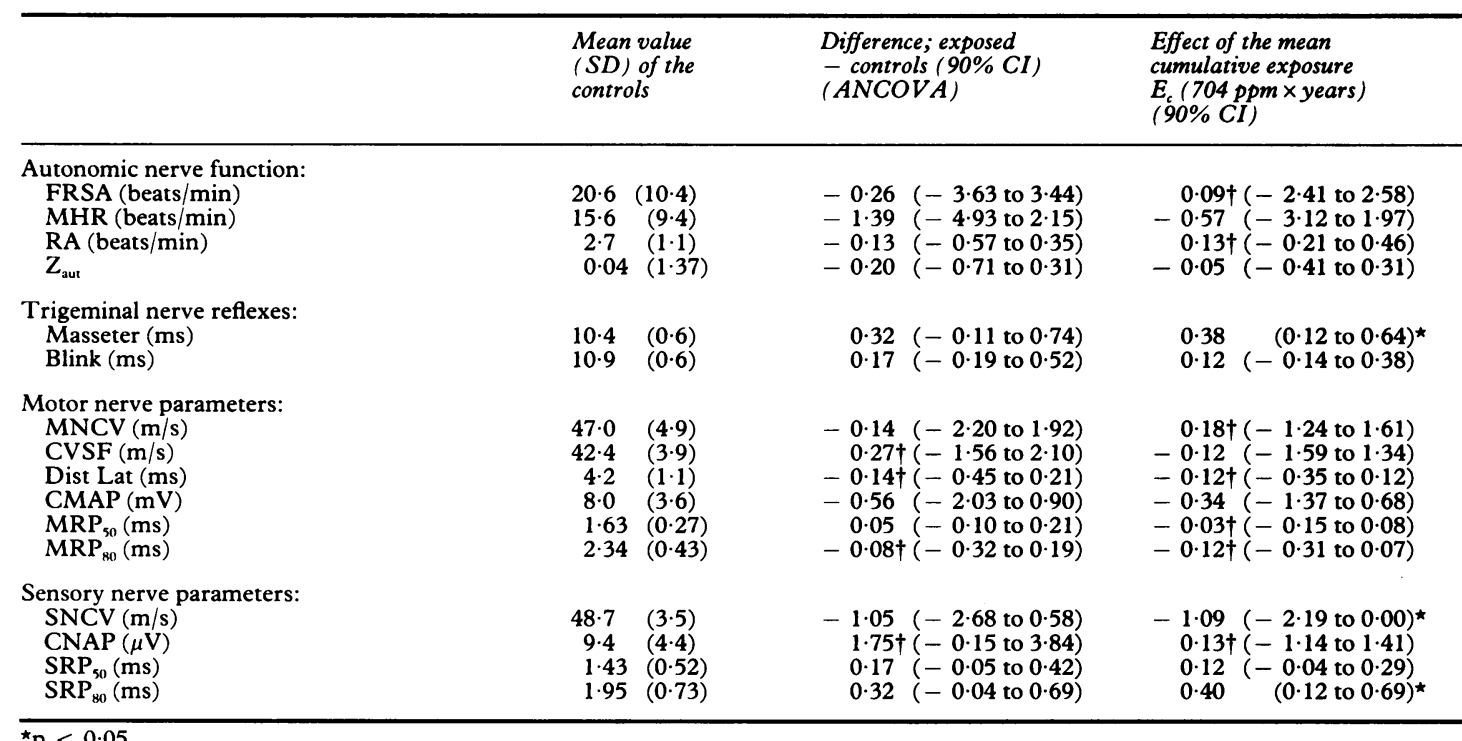

${ }^{\star} \mathrm{p}<0.05$.

+Effects in the non-pathological direction.

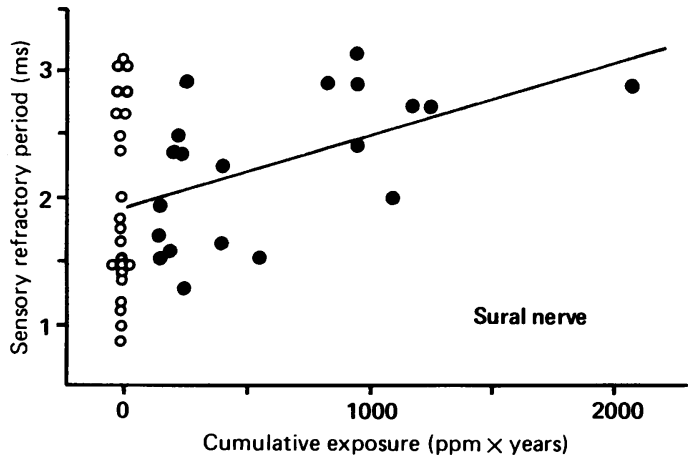

Sensory refractory period $\left(S R P_{80}\right)$ after correction for covariables against cumulative exposure $\left(E_{c}\right)$; the figure refers to 20 printers exposed to trichloroethene and 23 controls.

trols on a group basis (ANCOVA) no effects were detected (table).

The mean score of complaints in the questionnaire- 16 was 2.6 for the exposed workers and 1.8 for the controls ( $p>0 \cdot 10$, test of Yates and Cochran). Hogstedt $e t a l^{13}$ state that in persons over 28 years of age a score of seven or more indicates further examination; five exposed workers and one control met this criterion. Statistical testing showed that this difference almost reached significance (Fisher's exact test: $\mathrm{p}=0 \cdot 10$ ).

\section{Discussion}

\section{EXPOSURE}

It is likely that there will have been some difference in exposure between different jobs. Personal air samples would have provided a more precise indication of exposure than spot samples with gas detection tubes. All workers had been employed as printers in the same workplace and factory, however, and this probably indicates a higher homogeneity of exposure within this group than any previous investigation regarding TRI, where workers from several plants and occupations were examined. ${ }^{4567}$ Furthermore the exposure data together with the information on the process and the hygienic conditions supported the assumption of a constant concentration in the past up until the installation of the exhaust 11 years before the conduct of this study. We consider the available exposure data feasible to calculate a cumulative exposure $\left(E_{c}\right)$. We even attach more value to the results taking $\mathrm{E}_{\mathrm{c}}$ into account, because we consider this more logical than completely disregarding differences in exposure.

The statistical model used possesses some characteristics that are important for the interpretation of the results. Firstly any random error in predictor variables (like exposure) will attenuate the value of the calculated effects. Secondly it is impossible to calculate a no effect exposure. The possible existence of a mechanism of repair for neural impairment may complicate the interpretation of the results: part of the effect may have recovered during the past three years with decreasing exposure. We formerly solved this problem by weighting the past exposure. ${ }^{12}$ Our present exposure data do not allow such handling, however. If (partial) recovery has occurred the magnitude of the effect is underestimated. Because of the slow rate of recovery of most toxic neuropathies 
after long term exposure the results probably have not changed substantially during the past three years.

\section{EFFECTS}

We found no indication of an effect on the autonomic nerve system. As in a previous investigation ${ }^{12}$ the very large scatter is an important problem in the detection of an effect. This applies to the MHR in particular, where a number of subjects with a very low grip strength (despite proper encouragement) and an associated small response (down to zero) increased the scatter in the results. As a large scatter in the responses seems to be inherent to these methods ${ }^{912}$ their use in epidemiological research is limited unless large effects are expected or large populations can be studied.

The mean masseter reflex latency in the control group $(10.4 \mathrm{~ms})$ is somewhat longer than might be expected from other published data ${ }^{19}$; the reason is probably the mechanical delay caused by the rubber roller. We found a prolonged masseter reflex latency whereas this was not the case for the blink reflex latency. This difference may be explained by the functional characteristics and the nerve fibres involved in the reflex pathways. The afferent proprioceptive fibres concerned in the masseter reflex are thick and the reflex is monosynaptic; the efferent pathway runs through the trigeminal motor root. The afferent exteroceptive (nociceptive) fibres of the blink reflex are thinner than those in the masseter reflex and the reflex is polysynaptic; the efferent pathway runs through the facial nerve. Furthermore the reflexes are relayed in different parts of the trigeminal sensory nucleus. The difference in efferent pathways is in accordance with an explanation of the observed effects being concerned with selective vulnerability of the trigeminal nerve, but the other differences should be considered as well. The calculations were from $45 \%$ of the exposed workers and $53 \%$ of the controls. Because the decision of inclusion was blind and apparently independent with respect to exposure, it is unlikely that this selection has caused a bias. The significance to health of an increase in masseter reflex latency is not yet clear since the extent of prolongation of the latency time of this reflex in overt pathology is unknown. Barret et al $^{45}$ also examined trigeminal reflexes in workers exposed to TRI. Unfortunately, no description of their methodology nor reflex latencies are given, so their results cannot be compared with our data.

The changes in the motor functions of the peroneal nerve are marginal with relatively narrow confidence intervals. Even the CVSF, a sensitive indicator in many subclinical toxic neuropathies, had not decreased. An effect on this nerve can be excluded with reasonable certainty. In the sural nerve we found a small decrease in the SNCV and an increase in the $\mathrm{SRP}_{80}$. The calculation of the effect on the $\mathrm{SRP}_{80}$ concerned $64 \%$ of the exposed workers and $82 \%$ of the controls. An important reason for not being included in this calculation was if all response amplitudes were smaller than $80 \%$. The potentially resulting exclusion bias will be towards no effect, so it is likely that the true effect will be more pronounced. The prolongation of the $S_{R P}$ and not of the $S_{8 R} P_{50}$ suggests that the nerve fibres with a longer refractory period are more sensitive to TRI (or maybe to any neurotoxic factor) than those with shorter refractory periods. This trend even becomes clearer when the $\mathrm{SRP}_{0}$ is considered (the RP of the shortest refractory fibres, not in the table), which shows no effect at all, whereas the $\mathrm{SRP}_{50}$ tends towards the pathological direction. This may suggest that the conduction velocity of slower nerve fibres in the sural nerve is more impaired than the velocity of the fastest fibres, but only the last was measured.

Seppäläinen and Antti-Poika ${ }^{6}$ also measured nerve conduction, but no comparison can be made since they only give qualitative results. Triebig et al ${ }^{7}$ found no effect on conduction velocity in arm nerves in a group with about the same exposure level but a mean duration of exposure of only seven instead of 16 years. The small effect that we found fits in well, especially when the general higher susceptibility to neurotoxic substances of leg nerves is considered as compared with arm nerves.

Although no evidence was found for neuropsychiatric symptoms with the questionnaire-16, we cannot exclude mild symptoms; the applied method is too crude to warrant this interpretation. We think that the tendency towards an effect in this investigation calls for an evaluation with more subtle methods of an equally exposed group.

\section{HEALTH IMPLICATIONS}

With respect to the significance to health of the observed effects we consider these as changes in a negative direction that may reduce the reserve capacity to cope with other noxious influences. This reduction may bring nearer the moment at which functional deficits will be perceived. We consider the effects as undesirable, until it is established that these are not detrimental to health in the long term.

It is not possible to derive a no adverse effect level for occupational exposure to TRI from this study. One may try, however, to calculate by extrapolation the exposure level for lifetime (40 years) employment that would result in the observed effects on the basis of our model. The mean cumulative exposure of $704 \mathrm{ppm} \times$ years then leads to a predicted level of $704 / 40=17 \mathrm{ppm}$. If the mentioned effects are considered to be unacceptable a proportionally reduced exposure should be considered. It is also possible to calculate the effect of 40 years of exposure to the current threshold limit value, resulting in 
$1400 \mathrm{ppm} \times$ years cumulative exposure. Given the limited knowledge about the validity of our model and the limited accuracy of the exposure data these values should be considered merely as crude approximations.

We thank Mr P van der Peijl and Mr E Polak of the company's health department for the selection of the subjects and the organisation during the measurement period; we also thank Professor B W Ongerboer de Visser, Department of Clinical Neurophysiology, for his critical comments on earlier versions of this paper.

Requests for reprints to: $M$ Ruijten, Coronel Laboratory, Academical Medical Center, Meibergdreef 15, $1105 \mathrm{AZ}$ Amsterdam, The Netherlands.

1 Feldman RG, Mayer RM, Taub A. Evidence for peripheral neurotoxic effect of trichloroethylene. Neurology 1970; 20:599-606.

2 Mitchell ABS, Parsons-Smith BG. Trichloroethylene neuropathy. Brit Med J 1969;1:422-3.

3 Feldman RG. Trichloroethylene. In: Vinken PJ, Bruyn GW, eds. Handbook of clinical neurology, Vol 36: Intoxications of the nervous system, part I. Amsterdam: Elsvier, 1979:457-64.

4 Barret L, Faure J, Guillard B, et al. Trichloroethylene occupational exposure: elements for better prevention. Int Arch Occup Environ Health 1984;53:283-9.

5 Barret L, Garrel S, Danel V, et al. Chronic trichloroethylene intoxication: a new approach by trigeminal-evoked potentials? Arch Environ Health 1987;42:297-302.

6 Seppäläinen AM, Antti-Poika M. Time course of electrophysiological findings for patients with solvent poisoning; a descriptive study. Scand J Work Environ Health 1983;9: $15-24$.
7 Triebig G, Trautner P, Weltle D, et al. Untersuchungen zur Neurotoxizität von Arbeitsstoffen; III Messung der motorischen und sensorischen Nervenleitgeschwindigkeit bei beruflich Trichloräthylen-belasteten Personen. Int Arch Occup Environ Health 1982;51:25-34.

8 Spencer PS, Schaumburg HH. Organic solvent neurotoxicity; facts and research needs. Scand J Work Environ Health 1985;11 (suppl):53-60.

9 Matikainen E, Juntunen J. Examination of the peripheral autonomic nervous system in occupational neurology. In: Neurobehavioural methods in occupational and environmental health. Copenhagen: WHO/Euro, 1985:57-60.

10 Schütt P, Muche H, Lehmann HJ. Refractory period impairment in sural nerves of diabetics. J Neurol 1983;229:113-9.

11 Lowitsch K, Göhring U, Hecking E, et al. Refractory period, sensory conduction velocity and visual evoked potentials before and after haemodialysis. J Neurol Neurosurg Psychiatry 1981;44:121-8.

12 Ruijten MWMM, Sallé HJA, Verberk MM, Muijser H. Special nerve functions and colour discrimination in workers with long term low level exposure to carbon disulphide. Brit $J$ Ind Med 1990;47:589-95.

13 Hogstedt C, Andersson K, Hane M. A questionnaire approach to the monitoring of early disturbances in central nervous functions. In: Aitio A, Riihimaki V, Vaino V, eds. Biological monitoring and surveillance of workers exposed to chemicals. Washington: Hemisphere Publ 1984:275-87.

14 Ongerboer de Visser BW. Clinical uses of recorded jaw and blink reflexes. In: Struppler A, Weindl A, eds. Electromyography and evoked potentials. Berlin: Springer Verlag, 1985:146-53.

15 Faisst S, Meyer $M$. A non-invasive computerized measurement of motor neurone refractory period and subnormal conduction in man. Electroencephalogr Clin Neurophysiol 1981;51:548-58.

16 Kimura J. A method for estimating the refractory period of motor fibers in the human peripheral nerve. J Neurol Sci 1976;28:485-90.

17 Hopf HC. Untersuchungen über die Unterschiede in der Leitgeschwindigkeit motorischer Nerverfasern beim Menschen. Deutsche Zeitschrift für Nervenheilkunde 1962; 183:579-88.

18 Seppäläinen AM, Hernberg S. Sensitive technique for detecting subclinical lead neuropathy. Brit $J$ Ind Med 1972;29: 443-9.

19 Kimura J. The masseter reflex. In: Electrodiagnosis in diseases of nerve and muscle: principles and practice. Philadelphia: FA Davis company, 1989:361-3.

Accepted 20 August 1990 